\title{
Autocrine human growth hormone reduces mammary and endometrial carcinoma cell sensitivity to mitomycin $\mathbf{C}$
}

\author{
NICOLA M. BOUGEN ${ }^{1}$, TERESA YANG ${ }^{1}$, HELEN CHEN $^{1}$, PETER E. LOBIE $^{2}$ and JO K. PERRY ${ }^{1}$ \\ ${ }^{1}$ Liggins Institute, University of Auckland, Auckland 1023, New Zealand; ${ }^{2}$ Cancer Science Institute of Singapore \\ and Department of Pharmacology, National University of Singapore, Singapore 117456
}

Received February 23, 2011; Accepted April 4, 2011

DOI: 10.3892/or.2011.1305

\begin{abstract}
Drug resistance is a major cause of chemotherapy failure in breast cancer patients with metastatic disease. We previously demonstrated that autocrine human growth hormone $(\mathrm{hGH})$ plays a key role in oncogenic transformation and progression of mammary carcinoma. The present study investigated the role of autocrine $\mathrm{hGH}$ in the development of resistance to mitomycin $\mathrm{C}$ (MMC), an alkylating agent utilised in the treatment of advanced metastatic breast cancer. Stable forced expression of the $h G H$ gene was established in the mammary carcinoma cell lines MDA-MB-231, MCF-7 and T47D. Autocrine hGH reduced the sensitivity of mammary carcinoma cells to MMC in cell viability assays and reduced MMC-induced apoptotic cell death when compared to a control cell line. In addition, autocrine hGH enhanced MDA-MB-231 clonogenic survival, anchorage independent cell growth, growth in 3D Matrigel and protected MDA-MB-231 cells from induction of DNA double-strand breaks following MMC treatment. Functional antagonism of hGH in the endometrial carcinoma cell line RL95-2, which endogenously expresses hGH, significantly increased the sensitivity of these cells to MMC-induced DNA damage and cell death. Thus, autocrine hGH promotes mammary and endometrial carcinoma cell resistance to MMC. These studies indicate a potential role for antagonism of autocrine hGH in chemoresistant breast cancer.
\end{abstract}

\section{Introduction}

For patients with advanced metastatic breast cancer (MBC) treatment options are limited, as by this stage of the disease most patients are heavily pre-treated and resistant to commonly used chemotherapeutic agents. Therefore, the development of an effective chemotherapy regime for patients with $\mathrm{MBC}$

Correspondence to: Dr Jo K. Perry, The Liggins Institute, University of Auckland, 2-6 Park Avenue, Private Bag 92019, Auckland 1023, New Zealand

E-mail: j.perry@auckland.ac.nz

Key words: growth hormone, mammary carcinoma, endometrial carcinoma, chemotherapy, drug resistance, mitomycin C has become a clinical research focus. The alkylating agent mitomycin $\mathrm{C}$ (MMC) is a broad-spectrum chemotherapeutic which is able to inhibit tumour growth through promoting crosslinks between complementary strands of the DNA double helix resulting in DNA damage. This form of DNA lesion is so lethal that a single crosslink per genome is enough to cause bacterial cell death and 40 crosslinks are sufficient to kill a mammalian cell $(1,2)$. In its natural form MMC does not react with DNA, but is converted to an active form through reductive activation (3). Tumours provide a reasonably selective environment for MMC activation as both low $\mathrm{pH}$ and low oxygen stimulate the activation process (4). Consequently, MMC is selectively toxic for hypoxic solid tumours and suppresses their growth; this is referred to as cytostatic therapy $(4,5)$.

Bulky interstrand crosslinks (ICLs) constitute 5-13\% of the DNA adducts induced by MMC exposure (2). Similar to many other alkylating agents, MMC can cause a variety of DNA damage, such as double-strand breaks (DSBs), but ICLs are the major determinant of the lethality of these agents. However, it is widely accepted that DNA DSBs are formed as intermediates in the process of ICL repair $(6,7)$. Additional factors such as cellular uptake and metabolite activation also play a role in MMC efficacy. MMC is activated through cycles of reduction, which generates a number of reactive oxygen species (ROS), which can lead to additional DNA damage (8). The DNA lesions caused by MMC can lead to a number of outcomes such as selective inhibition of DNA synthesis and signal transduction, and ultimately apoptosis (8).

MMC is used clinically to treat a variety of solid tumours including that of the breast, the bladder and the upper gastrointestinal tract (9). MMC is administered to breast cancer patients intravenously and is used in combination with other chemotherapeutics and/or radiotherapy (10). In advanced breast cancer the most efficient use of MMC has been found to be in combination with vinca alkaloid drugs (9). The 'MV protocol' is frequently utilised as second line salvage therapy for advanced MBC. This involves the administration of MMC and vinca alkaloids, such as vinorelbine and vinblastine, and doses are tailored depending on the toxicities experienced by the patient $(11,12)$. MMC and vinca alkaloids have different mechanisms of action and have been found to exhibit antineoplastic synergy in vitro (13). A number of studies have also tested the efficacy of administration of MMC with doxorubicin 
in combination with other drugs, such as vinblastine (14); however, results have been less than promising. Preclinical studies demonstrated that MMC synergises with irinotecan, a topoisomerase I (TOPO I) inhibitor, most likely due to the ability of MMC to upregulate TOPO I expression and activity (15). In a phase II study of $32 \mathrm{MBC}$ patients pre-treated with taxane and anthracycline, sequential treatment with low dose MMC and irinotecan demonstrated a partial response in $31 \%$ of patients (16). However, MMC easily induces drug resistance (5), and its usage is often accompanied by severe side effects such as renal toxicity (6).

The oncogenic potential of autocrine human growth hormone $(\mathrm{hGH})$ in breast cancer has been previously demonstrated $(17,18)$. Autocrine hGH has been demonstrated to increase cell proliferation, survival and oncogenicity, and to increase migration and invasion of human mammary and endometrial carcinoma cells (17-23). Critically, autocrine hGH enhances tumour growth in xenograft models of human mammary (18) and endometrial carcinoma (21). Recent studies have demonstrated that autocrine $\mathrm{hGH}$ reduces sensitivity to the chemotherapeutic drugs doxorubicin, daunorubicin and tamoxifen $(17,24,25)$. In the present study, we investigated whether autocrine hGH confers resistance to MMC in mammary and endometrial carcinoma cells in vitro.

\section{Materials and methods}

Cell lines and transfection. MDA-MB-231, T47D, MCF-7 and RL95-2 cell lines were obtained from the American Type Culture Collection (ATCC). MDA-MB-231, T47D and MCF-7 cells were cultured at $37^{\circ} \mathrm{C}$ in $5 \% \mathrm{CO}_{2}$ in RPMI (Gibco) supplemented with $10 \%$ heat-inactivated FBS, $100 \mathrm{U} / \mathrm{ml}$ penicillin, $100 \mu \mathrm{g} / \mathrm{ml}$ streptomycin and $2 \mathrm{mM} \mathrm{L-glutamine}$. The RL95-2 wild-type cell line was cultured at $37^{\circ} \mathrm{C}$ in $5 \%$ $\mathrm{CO}_{2}$ in Advanced DMEM/F-12 (Invitrogen, Carlsbad, CA) supplemented with $10 \%$ heat-inactivated FBS, $100 \mathrm{U} / \mathrm{ml}$ penicillin, $100 \mu \mathrm{g} / \mathrm{ml}$ streptomycin and $2 \mathrm{mM}$ L-glutamine.

MDA-MB-231 and T47D cells were stably transfected with an expression plasmid containing the $h G H$ gene (pcDNA3$\mathrm{hGH}$ ) under the control of the CMV promoter (cell lines designated as MDA-hGH and T47D-hGH) using FuGENE 6 (MDA-MB-231 cell line) or FuGENE HD (T47D cell line) (Roche). For control purposes, cells were stably transfected with the empty pcDNA3 vector (cell lines designated as MDA-vec and T47D-vec). Stable transfectants were selected using $1200 \mathrm{mg} / \mathrm{ml}$ (MDA-MB-231) or $600 \mathrm{mg} / \mathrm{ml}$ (T47D) G418 over 21 days. Stable transfection of MCF-7 cells with pcDNA3-hGH has been described previously (26). These cells are designated MCF7-vec and MCF7-hGH.

For the assays investigating the effect of functional inhibition of hGH, B2036 (Pfizer, USA) was added to the medium (1000 nM). Bovine serum albumin (BSA) (Sigma Aldrich) was utilised as a protein control at equivalent concentrations.

Semi-quantitative RT-PCR. Total RNA was isolated from MCF7-vec and MCF7-hGH cell lines using TRIzol (Invitrogen). Semi-quantitative RT-PCR was performed using a OneStep RT-PCR kit (Qiagen, Valencia, CA). Sequences of the nucle- otide primers for RT-PCR were: hGH, 5'-CCGACACCCTCC AACAGGGA-3' and 5'-CCTTGTCCATGTCCTTCCTG-3'; hGHR, 5'-CTCAACTGGACTTTACTGAACG-3' and 5'-AATC TTTGGAACTGGAACTGGG-3'; $\beta$-actin, 5'-ATGATATCG CCGCGCTCG-3' and 5'-CGCTCGGTGAGGATCTTCA-3'. Amplified RT-PCR products were visualised on a $1.5 \%$ agarose gel.

Western blotting. Soluble whole cellular extracts were run on an SDS-PAGE and immunoblotted as previously described (27) using a rabbit anti-hGH antibody (National Hormone and Peptide Program, Torrance, CA) and anti-mouse secondary antibody conjugated with horseradish peroxidase (Upstate Biotechnology, Lake Placid, NY). Blots were stripped and reprobed by using a $\beta$-actin antibody (Santa Cruz Biotechnology) as a protein loading control. Protein bands were detected using the Phototype horseradish peroxidase Western blot detection system (SuperSignal West Dura extended duration substrate; Pierce, Rockford, IL).

Wst-1 viability assay. MDA-vec/MDA-hGH cells (5000) or RL95-2 wild-type cells were plated in 96 wells and cultured $24 \mathrm{~h}$ prior to treatments. For the MMC dose response curves, cells were treated with 0-40 $\mu \mathrm{M}$ (MDA-vec and MDA-hGH) or 0-5 $\mu \mathrm{M}$ (RL95-2) MMC (in full serum media) for $48 \mathrm{~h}$. Cell viability was measured using the Wst-1 reagent (Roche). Absorbance was read at 440 and $650 \mathrm{~nm}$.

Total cell number. Cells $\left(5 \times 10^{4}\right)$ were seeded into 6 -well plates in monolayers in full serum media. The media was replaced with serum-free media for $24 \mathrm{~h}$, followed by MMC (3 $\mu \mathrm{M})$ treatment. After $24 \mathrm{~h}$ of treatment, the cells were trypsinised with $0.5 \%$ trypsin, and the cell number was determined using a haemocytometer.

Apoptosis assay. Apoptotic cell death was measured by fluorescence microscopic analysis of cell DNA staining patterns with Hoechst 33258 as previously described (28). MDA-vec and MDA-hGH cells or RL95-2 wild-type cells were plated at $2 \times 10^{5}$ cells/well in full serum media in 6-well plates and cultured for $24 \mathrm{~h}$. Cells were washed with PBS, and the media replaced with serum-free media. After $1 \mathrm{~h}, 0.5 \mu \mathrm{M} \mathrm{MMC}$ was added to the media. After 24, 48 or $72 \mathrm{~h}$, the cells were fixed and permeabilised in $4 \%$ paraformaldehyde, $1 \%$ Triton X-100 and stained with $4 \mu \mathrm{g} / \mathrm{ml}$ of the karyophilic dye Hoeschst 33258 in PBS for $15 \mathrm{~min}$ at room temperature. Cells were washed with PBS, and apoptotic nuclear morphology was determined using an inverted UV fluorescence microscope (Olympus). For statistical analysis, at least 200 cells were counted in eight random microscopic fields at x400 magnification.

Cell survival clonogenic assay. Exponentially growing MDA-vec and MDA-hGH cells were pre-treated with $0.5 \mu \mathrm{M}$ MMC for $2 \mathrm{~h}$, replated at 800 cells/well in a 6-well plate and cultured in full serum media for 14 days. Colonies were stained with $0.1 \%$ crystal violet in $20 \%$ ethanol and counted. Colonies with $>50$ cells were counted, and the plating efficiencies (PE) were calculated. $\mathrm{PE}=$ (number of colonies formed/number of cells seeded) $\times 100 \%$. 
A
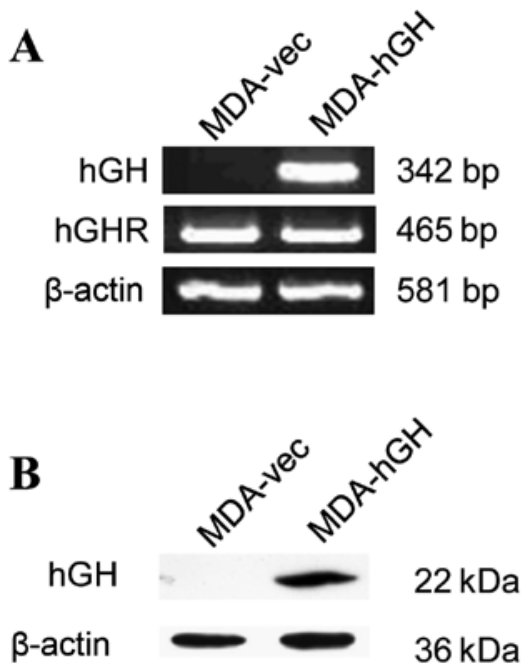
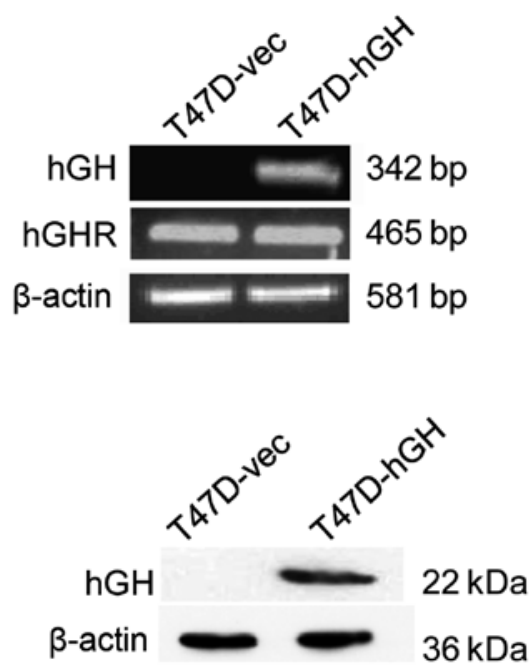

Figure 1. Forced expression of hGH in mammary carcinoma cells. Mammary carcinoma cell lines MDA-MB-231 and T47D were stably transfected with an expression vector containing the human growth hormone $(h G H)$ gene (designated MDA-hGH and T47D-hGH, respectively). Control cells lines were transfected with the empty pCDNA3 vector (designated MDA-vec and T47D-vec, respectively). (A) The level of hGH and hGH receptor (hGHR) mRNA in stably transfected MDA-MB-231 and T47D cells was determined by semi-quantitative RT-PCR. $\beta$-actin was used as a loading control. (B) Western blot analysis was used to assess hGH protein levels in the stably transfected mammary carcinoma cells. $\beta$-actin was used as a loading control.

Colony formation in soft agar. A soft agar assay was performed as previously described (18). Briefly, untreated or MMC-treated $(0.5 \mu \mathrm{M}, 24 \mathrm{~h}) \mathrm{MDA}-\mathrm{vec}$ and MDA-hGH cells were suspended in $0.35 \%$ agarose (in $10 \%$ serum media) and plated in 6-well plates (5000 cells/well). After 14 days in culture, the colonies were stained with crystal violet and counted.

3D Matrigel. A 3D Matrigel assay was performed as previously described (29). Briefly, MDA-vec and MDA-hGH or RL95-2 cells were plated at 1000 cells per well in $4 \%$ growth factorreduced Matrigel ${ }^{\mathrm{TM}}$ (BD Biosciences) and 5\% serum media in 96-well plates and allowed to form colonies. On day 4, RL95-2 cells were treated with $1000 \mathrm{nM}$ BSA or B2036. After $24 \mathrm{~h}$, the cells were treated with $0.5 \mu \mathrm{M}$ MMC. On day 9 , cell viability was determined using the Wst-1 reagent (Roche).

Neutral comet assay. A neutral comet assay was performed as previously described (30). Briefly, MDA-vec and MDA-hGH cells were treated with $3 \mu \mathrm{M}$ MMC for $2 \mathrm{~h}$ and embedded in low melting temperature Seaplaque Agarose (Cambrex Bio Science) on GelBond film (Lonza Rockland, Inc.). The cells were lysed overnight at $37^{\circ} \mathrm{C}$ in neutral lysis solution $(2 \%$ sarkosyl, $0.5 \mathrm{M} \mathrm{Na}_{2}$ EDTA, $0.5 \mathrm{mg} / \mathrm{ml}$ proteinase $\mathrm{K}, \mathrm{pH} 8.0$ ) and then washed in rinse buffer $(90 \mathrm{mM}$ Tris buffer, $90 \mathrm{mM}$ boric acid, 2 mM Na 2 EDTA, pH 8.5) 3 times. Slides were subjected to electrophoresis in $1 \mathrm{X}$ TBE for $25 \mathrm{~min}$ at $20 \mathrm{~V}$. Comets were stained with $10 \mu \mathrm{g} / \mathrm{ml}$ propidium iodide for $20 \mathrm{~min}$ and rinsed in $400 \mathrm{ml}$ distilled water to remove excess stain. At least 100 comet images from each slide were examined. Comet tail length and tail moment were analysed using Tritek CometScore software (version 1.5).

Statistics. All numerical data are expressed as mean \pm SEM of triplicate determinants. Statistical significance between treatment groups was determined using an unpaired twotailed t-test or a two-way analysis of variance (ANOVA; Bonferroni) using SigmaPlot 11.0. All experiments were repeated at least 3 times. A single representative figure is shown.

\section{Results}

Autocrine $h G H$ reduces mammary carcinoma cell sensitivity to treatment with $M M C$. To determine whether autocrine hGH reduces sensitivity to MMC we stably transfected MDA-MB-231 and T47D cells with an expression vector containing the $h G H$ gene (MDA-hGH and T47D-hGH). For control purposes, parental cells were also stably transfected with the empty pcDNA3 vector (MDA-vec and T47D-vec). Forced expression of hGH in the MDA-hGH and T47D-hGH cells was verified at both the mRNA (Fig. 1A) and protein (Fig. 1B) levels compared with the respective control cell lines (MDA-vec and T47D-vec). Forced expression of hGH in MCF-7 cells has been described previously (26).

A dose response experiment measuring MDA-vec and MDA-hGH cell viability after treatment with MMC was performed in full serum media. At doses of MMC ranging from 1 to $40 \mu \mathrm{M}$, the MDA-hGH cells exhibited significantly higher cell viability than the MDA-vec cells ( $\mathrm{p}<0.001$; two-way ANOVA) (Fig. 2A). After treatment with $10 \mu \mathrm{M} \mathrm{MMC}$, the MDA-hGH cells exhibited $17 \%$ higher cell viability after $38 \mathrm{~h}$ than the MDA-vec cells ( $\mathrm{p}<0.001)$. Total cell number assays were performed in hGH-transfected stable cell lines derived from MDA-MB-231, T47D and MCF-7 mammary carcinoma cells after $24 \mathrm{~h}$ of MMC treatment in serum-free media. Autocrine hGH significantly reduced sensitivity to MMC treatment in all three cell lines. Following MMC treatment MDA-hGH, T47D-hGH and MCF7-hGH cells exhibited 41, 36.3 and $34.5 \%$ higher total cell number when compared with the respective control transfected cells, MDA-vec, T47D-vec and MCF7-vec (Fig. 2B).

In an apoptosis assay, autocrine hGH protected MDA-MB231 cells from apoptotic cell death induced by serum deprivation (Fig. 2C). In addition, autocrine hGH protected 
A

\section{Cell Viability}

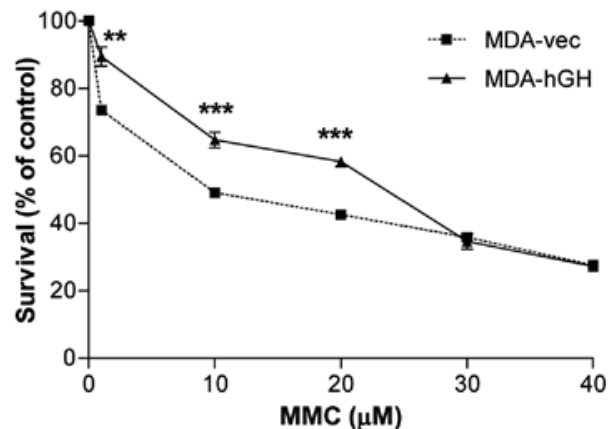

B

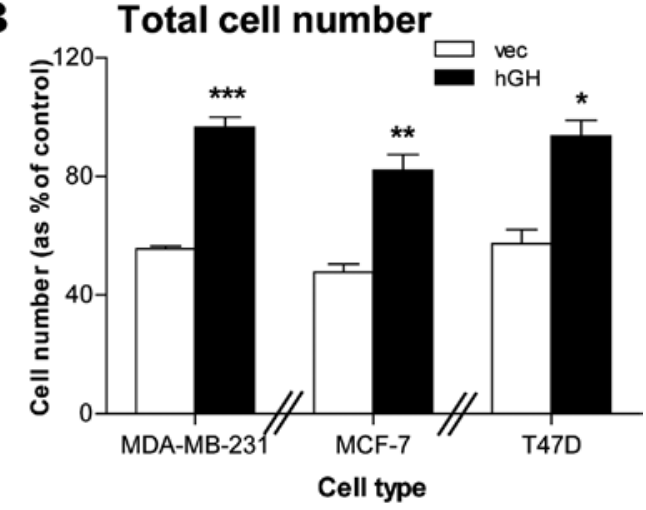

$\mathbf{C}$

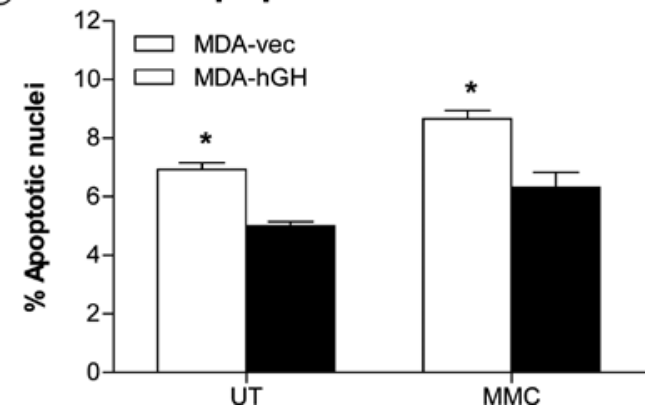

Figure 2. Autocrine hGH enhances mammary carcinoma cell viability and survival after treatment with MMC. (A) MDA-vec and MDA-hGH cells were cultured in full serum medium (10\% FBS). A Wst-1 assay was performed $48 \mathrm{~h}$ after treatment with MMC $(0-40 \mu \mathrm{M})$. (B) A total cell number assay was performed $24 \mathrm{~h}$ after treatment of MDA-vec/MDA-hGH, MCF7-vec/ MCF7-hGH or T47D-vec/T47D-hGH cells with $3 \mu \mathrm{M}$ MMC in serum-free medium. The two diagonal lines indicate that each pair of stably transfected cell lines was investigated in separate experiments. (C) Apoptosis assay. MDA-vec and MDA-hGH cells were treated with $0.5 \mu \mathrm{M}$ MMC in serumfree media, and apoptosis was quantified $48 \mathrm{~h}$ later.

against apoptosis induced by MMC treatment in serum-free media. MMC-mediated induction of apoptotic cell death was $40 \%$ higher in MDA-vec cells when compared with MDA-hGH cells $(\mathrm{p}<0.05)$ (Fig. 2C).

Autocrine hGH enhances clonogenic survival, anchorage independent growth and protects against DNA damage following MMC treatment. A clonogenic survival assay was used to measure the ability of MDA-vec and MDA-hGH cells to form colonies after MMC treatment. There was no significant difference in plating efficiency (PE) between the untreated MDA-vec and MDA-hGH cells (two-way ANOVA). However, after treatment with $0.5 \mu \mathrm{M} \mathrm{MMC}$, the PE for
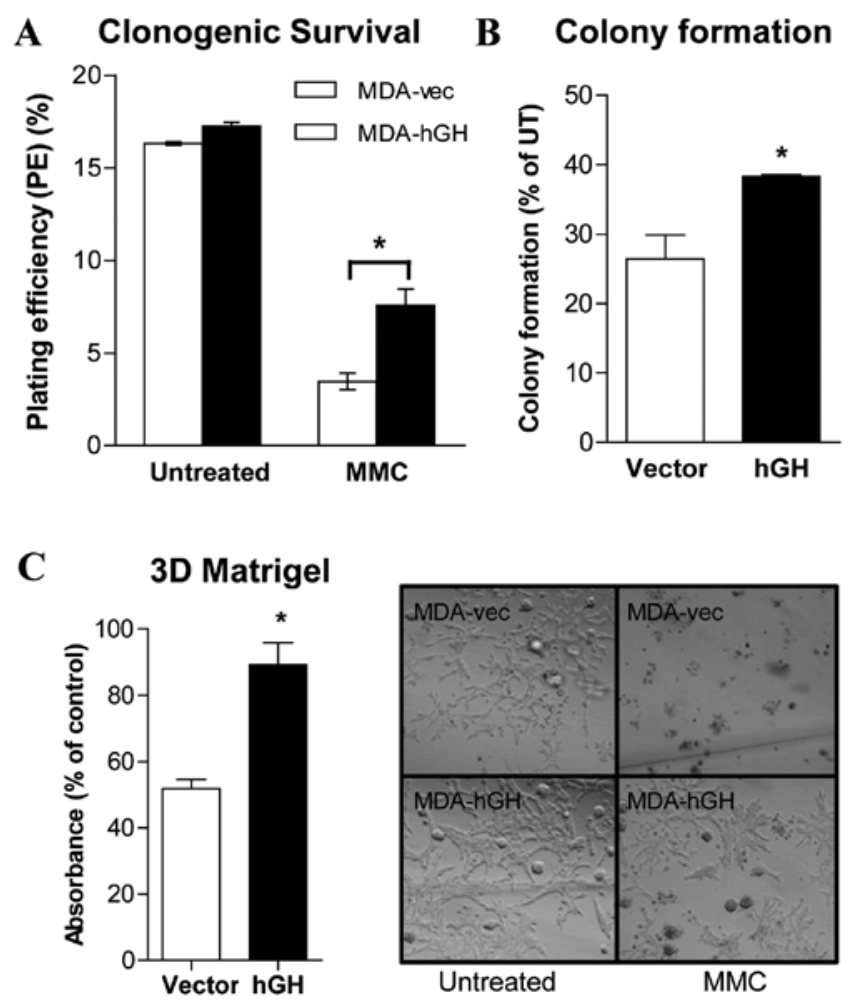

D

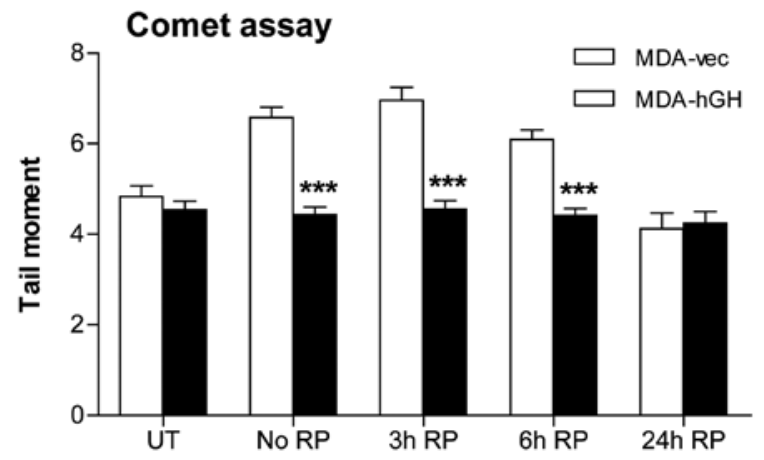

Figure 3. Autocrine hGH increases clonogenic survival, colony formation, growth in Matrigel, and protects against DNA damage following MMC treatment. (A) Clonogenic assay. Untreated or MMC-treated $(0.5 \mu \mathrm{M}, 2 \mathrm{~h})$ cells were plated at 800 cells/well and cultured for 14 days. Colonies of $>50$ cells were counted, and plating efficiencies (PE) were calculated. (B) Soft agar assay. MMC-treated cells were embedded in agarose and cultured for 14 days, after which colonies were stained with crystal violet and counted. (C) 3D Matrigel assay. MDA-vec and MDA-hGH cells were embedded in $4 \%$ Matrigel $^{\mathrm{TM}}$ and cultured for 4 days prior to treatment with $0.5 \mu \mathrm{M}$ MMC. Colonies were quantified with Wst-1 on day 9. Two-way ANOVA tests (Bonferroni) indicated a significant reduction in sensitivity to $\mathrm{MMC}$ in MDA-hGH cells when compared to the MDA-vec cell line $(\mathrm{A}-\mathrm{C})(\mathrm{P}<0.05)$. (D) Neutral comet assay. After $2 \mathrm{~h}$ of treatment with $0.5 \mu \mathrm{M} \mathrm{MMC}$, DNA double-strand breaks were quantified in MDA-vec and MDA-hGH cells over a 24-h period $\left({ }^{*} \mathrm{P}<0.05\right.$ and $\left.{ }^{* * *} \mathrm{P}<0.001, \pm \mathrm{SEM}\right)$.

MDA-hGH cells was 2.1-fold higher than MDA-vec cells ( $<<0.05$; two-way ANOVA) indicating that autocrine hGH enhanced MDA-MB-231 clonogenic survival following treatment with MMC (Fig. 3A).

Autocrine hGH has previously been demonstrated to enhance anchorage independent growth in MCF-7 cells (18). In the present study autocrine hGH increased the formation of colonies by MDA-MB-231 cells in a soft agar assay (Fig. 3B). Following treatment with MMC, autocrine hGH enhanced 
A

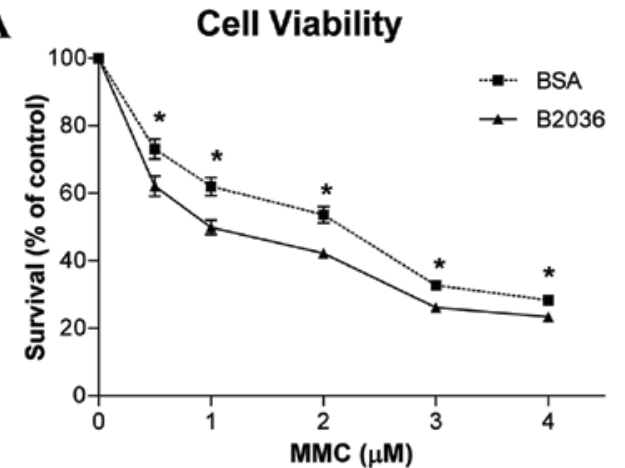

B Apoptosis

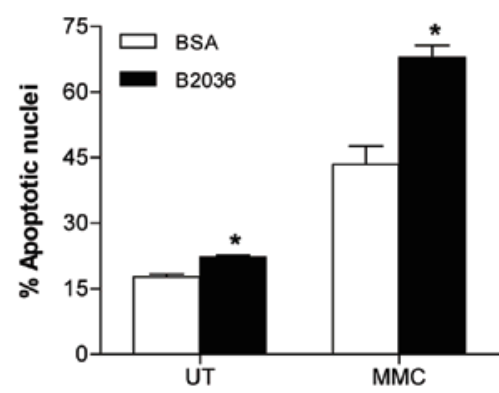

C 3D Matrigel

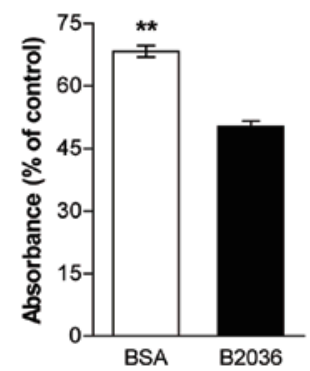

D

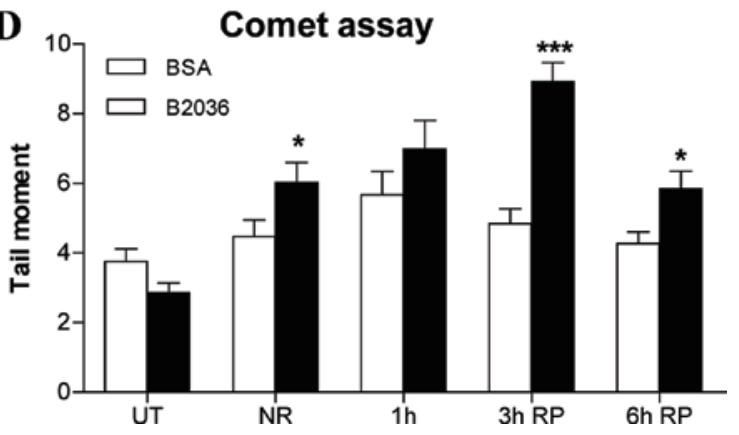

Figure 4. Functional inhibition of hGH reduces endometrial cell proliferation and growth in 3D Matrigel, while increasing apoptotic cell death and DNA damage following MMC treatment. (A) Cell viability. RL95-2 wild-type cells were cultured in full serum medium (10\% FBS), treated with BSA or B2036 $(1000 \mathrm{nM})$, and a Wst-1 assay was performed at $48 \mathrm{~h}$ after treatment with MMC $(0-5 \mu \mathrm{M})$. (B) Apoptosis assay. BSA- or B2036-treated RL95-2 cells were grown in serum-free medium, and apoptosis was quantified $48 \mathrm{~h}$ after treatment with $0.5 \mu \mathrm{M}$ MMC. (C) 3D Matrigel assay. RL95-2 cells were grown in 4\% Matrigel $^{\mathrm{TM}}$ (diluted with 5\% FBS medium) for 4 days and pretreated with BSA or B2036 for 24 h followed by $0.5 \mu \mathrm{M}$ MMC on day 5 . Colony formation was examined by light microscopy and quantified using Wst-1 on day 9. (D) Neutral comet assay. After a 2-h treatment wih 0.5 $\mu$ M MMC, DNA double-strand breaks were quantified in cells treated with BSA and B2036 over a 6-h period.

colony formation by MDA-hGH cells 1.45 -fold compared to the MDA-vec cells (p<0.05; two-way ANOVA) (Fig. 3B).

MDA-vec and MDA-hGH stable cell lines were cultured in the reconstituted basement membrane extract, Matrigel, following treatment with MMC. In a 3D Matrigel assay, autocrine hGH enhanced cell viability following MMC treatment (Fig. 3C). MDA-vec and MDA-hGH cells were grown in $4 \%$ Matrigel and treated for 4 days with MMC. Cells were examined using light microscopy, and the cell viability was quantified using the Wst-1 reagent. In both the untreated and MMC-treated wells, the MDA-hGH cells formed larger and more invasive colonies when compared to the MDA-vec cells (Fig. 3C). After MMC treatment, a 1.72-fold increase in cell viability in the MDA-hGH cells was observed compared with the MDA-vec control cell line ( $<<0.05$; two-way ANOVA) (Fig. 3C).

Autocrine hGH previously was found to protect mammary and endometrial carcinoma cells from DNA damage following exposure to ionising radiation (Bougen et al, unpublished data). We, therefore, hypothesised that reducing DNA damage may also be one mechanism whereby autocrine hGH protects cells against MMC-induced cell death. A neutral comet assay, which detects DNA DSBs, was utilised to examine any differences in DNA DSB induction after MMC treatment in MDA-vec and MDA-hGH cells. No significant difference was observed in endogenous DNA DSB levels between the untreated MDA-vec and MDA-hGH cells. Following MMC treatment, the MDA-vec cells exhibited a significant increase in tail moment (defined as the product of the tail length and the fraction of total DNA in the tail) which was sustained for $6 \mathrm{~h}$ after treatment and returned to baseline levels after $24 \mathrm{~h}$ (Fig. 3D). In contrast, no significant increase in tail moment was observed in the MDA-hGH cells after MMC treatment at any time point examined (Fig. 3D). Thus, autocrine hGH protected MDA-MB-231 cells from DNA DSBs induced by MMC treatment.

Functional inhibition of autocrine $h G H$ sensitises $R L 95-2$ endometrial carcinoma cells to MMC-mediated cell death and DNA damage. Although MMC is not routinely used to treat endometrial cancer, RL95-2 cells express hGH endogenously (21) and provide a useful model to investigate the consequences of functional antagonism of hGH on MMC-induced DNA damage and repair. Functional antagonism of hGH was achieved using the hGH-receptor antagonist, B2036. B2036 is the protein component of Pegvisomant ${ }^{\circledR}$ (Pfizer) which is FDA approved for the treatment of acromegaly $(31,32)$. To determine whether functional inhibition of hGH sensitises RL95-2 cells to MMC, a MMC dose response experiment was performed with RL95-2 cells pretreated with B2036. Cells were pretreated with either 1000 nM B2036 or BSA (as a control) for $24 \mathrm{~h}$ prior to treatment with MMC in full serum media. B2036 enhanced the effect of MMC treatment at all doses tested (Fig. 4A).

Functional inhibition of hGH utilising B2036 was previously found to significantly increase RL95-2 apoptotic cell death in serum-free conditions (21). To determine whether B2036 treatment enhanced RL95-2 apoptotic cell death following MMC 
treatment, cells were pre-treated with B2036 for $24 \mathrm{~h}$ and then treated with MMC. Treatment with MMC increased apoptotic cell death in the B2036-treated RL95-2 cells from 22.3 to $67.9 \%$ (3.1-fold increase) whereas in the control BSA-treated cells, MMC increased apoptotic cell death from 17.7 to $43.5 \%$ (2.45-fold increase) (Fig. 4B). Furthermore, in a 3D Matrigel assay, pre-treatment of RL95-2 cells with B2036 reduced cell growth after MMC treatment by 1.4 -fold (p<0.001) (Fig. 4C).

In a neutral comet assay, B2036 significantly enhanced induction of DNA DSBs after treatment with MMC compared with the BSA-treated controls (Fig. 4D). Treatment with B2036 enhanced MMC-induced DNA damage in RL95-2 cells at all time points examined (Fig. 4D). DNA damage was maximal at $3 \mathrm{~h}$ in the B2036-treated cells compared with $1 \mathrm{~h}$ in the control cells. The level of DNA DSBs returned to baseline following $6 \mathrm{~h}$ repair in control cells pre-treated with BSA. In contrast, levels of MMC-induced DNA DSBs had not returned to baseline levels at $6 \mathrm{~h}$ in cells pre-treated with B2036 (Fig. 4D). Thus, functional inhibition of hGH increases the sensitivity of RL95-2 cells to treatment with MMC.

\section{Discussion}

The primary cause of failure to chemotherapeutic treatment is drug resistance in tumour cells (33). Chemoresistance may be intrinsic or acquired and is most evident in advanced metastatic disease $(33,34)$. Multiple mechanisms of drug resistance have been proposed including pharmacological factors, protection from induced apoptosis, an enhanced cellular capacity to repair DNA damage or to tolerate oxidative cell stress, and overexpression of drug efflux pumps thereby reducing intracellular drug concentration $(33,35,36)$. Chemoresistance is a significant obstacle in the treatment of MBC, as patients are frequently heavily treated with a number of classes of chemotherapeutic agents and may have acquired resistance to multiple agents. As a second line, or salvage therapy, MMC is an attractive prospect for treating recurrent metastatic breast cancer as it is rarely used as a first line treatment, and the mechanisms of resistance to MMC have been demonstrated to be different to that of frequently used drugs such as doxorubicin and paclitaxel (37). A number of studies have demonstrated that MMC monotherapy elicits positive responses in doxorubicin-resistant patients (38-41).

Herein, this study demonstrated that autocrine hGH reduces MDA-MB-231 cell sensitivity to the alkylating agent MMC. This may occur through reduced apoptotic cell death and through reducing DNA damage induced by MMC. In the present study we observed that autocrine hGH protected MDA-MB-231 cells from MMC-induced apoptotic cell death. This is consistent with numerous studies demonstrating that autocrine hGH promotes cell survival $(18,20,23,27,42-45)$. We also observed that autocrine hGH protected MDA-MB-231 cells from MMC-induced DNA damage, while functional inhibition of autocrine hGH in RL95-2 cells increased MMC-induced DNA damage. Although MMC is not routinely used to treat endometrial cancer, RL95-2 cells express hGH endogenously and provide a useful model to investigate the consequences of functional antagonism of $\mathrm{hGH}$ on MMC-induced DNA damage and repair. We also recently demonstrated that autocrine hGH protects MDA-MB-231,
T47D and RL95-2 cells from DNA damage following exposure to ionising radiation treatment (Bougen et al, unpublished data). These studies suggest that autocrine hGH may confer resistance to DNA damaging agents by reducing the induction of DNA double-strand breaks.

One mechanism by which autocrine hGH confers protection against DNA damage may be through the increase in cellular antioxidant capacity. In addition to the generation of DNA-interstrand crosslinks, MMC generates free radicals when metabolised and promotes antitumour activity via ROS-dependant activation of apoptotic cell death and through the promotion of oxidative damage of DNA, including strand breaks and nucleotide modifications $(2,8)$. Although oxidative cell damage was not investigated in the present study, it has previously been demonstrated that autocrine hGH protects from oxidative stress-induced cell death in MCF-7 cells through regulation of a number of key enzymes involved in the cellular oxidative stress response, including catalase and superoxide dismutase 1 (42). Given that DNA damage and increased cellular oxidative stress are key effector mechanisms of specific chemotherapeutic agents, it is likely that antagonism of autocrine hGH will improve the efficacy of other chemotherapeutic regimes utilised for human mammary carcinoma.

$\mathrm{hGH}$ has also been implicated in the development of resistance to a number of other cytotoxic drugs $(24,25)$. Autocrine hGH reduces MCF-7 cell sensitivity to tamoxifen treatment in vitro (24). This may occur through upregulated expression of the estrogen receptor GPR30 $(24,43)$. GPR30 has been identified as a putative tamoxifen-resistance gene as it is not blocked by tamoxifen, unlike estrogen receptor $\alpha$; paradoxically tamoxifen acts as an agonist to GPR30 signalling $(43,44)$. Autocrine hGH also protects MCF-7 cells from cell death mediated by a number of other cytotoxic agents, including the anthracycline chemotherapeutic agent daunorubicin (42) and doxorubicin (25), while exogenous hGH enhances MCF-7 cell viability and DNA synthesis after doxorubicin treatment (25). Conversely, functional inhibition of hGH was found to restore sensitivity to doxorubicin in MCF-7 cells treated with exogenous hGH (25).

The treatment of cancer is becoming progressively more targeted, with the emergence of small-molecule and antibody treatments. There is increasing evidence that autocrine hGH, not only plays a role in the progression of breast cancer, but that it also confers resistance to multiple classes of chemotherapeutic agents including anti-estrogens and anthracyclines $(21,24,25,42)$. Thus, a combination of strategies that functionally inhibits hGH, in conjunction with conventional chemotherapeutic agents, may enhance the efficacy of treatment regimes for breast and endometrial cancers.

\section{Acknowledgements}

This study was funded by The New Zealand Breast Cancer Foundation (NZBCF), The Breast Cancer Research Trust and The Foundation of Science and Technology, New Zealand.

\section{References}

1. Tomasz M: Mitomycin C: small, fast and deadly (but very selective). Curr Biol 2: 575-579, 1995. 
2. Dronkert ML and Kanaar R: Repair of DNA interstrand crosslinks. Mutat Res 486: 217-247, 2001.

3. Mao Y, Varoglu M and Sherman DH: Molecular characterisation and analysis of the biosynthetic gene cluster for the antitumor antibiotic mitomycin C from Streptomyces lavendulae NRRL 2564. Chem Biol 6: 251-263, 1999.

4. Spanswick VJ, Cummings J and Smyth JF: Current issues in the enzymology of mitomycin C activation. Gen Pharmacol 31: 539-544, 1998

5. Sartorelli AC and Rockwell S: Mitomycin C: a prototype bioreductive agent. Oncol Res 6: 501-508, 1994.

6. Rothfuss A and Grompe M: Repair kinetics of genomic interstrand DNA cross-links: evidence for DNA double-strand break-dependent activation of the Fanconi anemia/BRCA pathway. Mol Cell Biol 24: 123-134, 2004.

7. Niedernhofer LJ, Odijk H, Budzowska M, et al: The structurespecific endonuclease Erccl-Xpf is required to resolve DNA interstrand cross-link-induced double-strand breaks. Mol Cell Biol 24: 5776-5787, 2004

8. Tomasz $\mathrm{M}$ and Palom Y: The mitomycin bioreductive antitumor agents: cross-linking and alkylation of DNA as the molecular basis of their activity. Pharmacol Ther 76: 73-87, 1997.

9. Bradner WT: Mitomycin C: a clinical update. Cancer Treat Rev 27: 35-50, 2001

10. Henderson IC: Recent advances in the usage of mitomycin. Oncology 50 (Suppl 1): 1-83, 1993.

11. Scheithauer W, Kornek G, Haider K, et al: Effective second line chemotherapy of advanced breast cancer with navelbine and mitomycin C. Breast Cancer Res Treat 26: 49-53, 1993.

12. Garewal HS, Brooks RJ, Jones SE and Miller TP: Treatment of advanced breast cancer with mitomycin $\mathrm{C}$ combined with vinblastine or vindesine. J Clin Oncol 1: 772-775, 1983.

13. Pouillart R, Huong TH, Brugerie E and Lheritier J: Sequential administration of two oncostatic drugs: study of modalities for pharmacodynamic potentiation. Biomedicine 21: 471-479, 1974.

14. Sulkes A, Gez E, Pfeffer MR, Catane R, Isacson R and Biran S Adriamycin, vinblastine and mitomycin $C$ as second-line chemotherapy in advanced breast cancer. Cancer Chemother Pharmacol 18: 162-167, 1986.

15. Xu Y and Shapiro CL: Rationale for mitomycin and irinotecan use in advanced breast cancer. Oncology 17: 25-28, 2003.

16. Mrozek E, Kolesar J, Young D, Allen J, Villalona-Calero M and Shapiro CL: Phase II study of sequentially administered low-dose mitomycin-C (MMC) and irinotecan (CPT-11) in women with metastatic breast cancer (MBC). Ann Oncol 19: 1417-1422, 2008

17. Perry JK, Mohankumar KM, Emerald BS, Mertani HC and Lobie PE: The contribution of growth hormone to mammary neoplasia. J Mammary Gland Biol Neoplasia 13: 131-145, 2008.

18. Zhu T, Starling-Emerald B, Zhang X, et al: Oncogenic transformation of human mammary epithelial cells by autocrine human growth hormone. Cancer Res 65: 317-324, 2005.

19. Kaulsay KK, Mertani HC, Tornell J, Morel G, Lee KO and Lobie PE: Autocrine stimulation of human mammary carcinoma cell proliferation by human growth hormone. Exp Cell Res 250 35-50, 1999.

20. Mukhina S, Liu D, Guo K, et al: Autocrine growth hormone prevents lactogenic differentiation of mouse mammary epithelial cells. Endocrinology 147: 1819-1829, 2006.

21. Pandey V, Perry JK, Mohankumar KM, et al: Autocrine human growth hormone stimulates oncogenicity of endometrial carcinoma cells. Endocrinology 149: 3909-3919, 2008.

22. Perry JK, Emerald BS, Mertani HC and Lobie PE: The oncogenic potential of growth hormone. Growth Horm IGF Res 16: 277-289, 2006.

23. Goh EL, Pircher TJ and Lobie PE: Growth hormone promotion of tubulin polymerization stabilizes the microtubule network and protects against colchicine-induced apoptosis. Endocrinology 139: 4364-4372, 1998.
24. Mojarrad M, Momeny M, Mansuri F, et al: Autocrine human growth hormone expression leads to resistance of MCF-7 cells to tamoxifen. Med Oncol 27: 474-480, 2010.

25. Zatelli MC, Minoia M, Mole D, et al: Growth hormone excess promotes breast cancer chemoresistance. J Clin Endocrinol Metab 94: 3931-3938, 2009.

26. Brunet-Dunand SE, Vouyovitch C, Araneda S, et al: Autocrine human growth hormone promotes tumor angiogenesis in mammary carcinoma. Endocrinology 150: 1341-1352, 2009.

27. Mertani HC, Zhu T, Goh EL, Lee KO, Morel G and Lobie PE: Autocrine human growth hormone $(\mathrm{hGH})$ regulation of human mammary carcinoma cell gene expression. Identification of $\mathrm{CHOP}$ as a mediator of hGH-stimulated human mammary carcinoma cell survival. J Biol Chem 276: 21464-21475, 2001

28. Kaulsay KK, Zhu T, Bennett W, Lee KO and Lobie PE: The effects of autocrine human growth hormone (hGH) on human mammary carcinoma cell behavior are mediated via the hGH receptor. Endocrinology 142: 767-777, 2001.

29. Amiry N, Kong X, Muniraj N, et al: Trefoil factor-1 (TFF1) enhances oncogenicity of mammary carcinoma cells. Endocrinology 150 4473-4483, 2009.

30. Olive PL and Banath JP: The comet assay: a method to measure DNA damage in individual cells. Nat Protoc 1: 23-29, 2006.

31. Kopchick JJ, Parkinson C, Stevens EC and Trainer PJ: Growth hormone receptor antagonists: discovery, development, and use in patients with acromegaly. Endocr Rev 23: 623-646, 2002.

32. van der Lely AJ and Kopchick JJ: Growth hormone receptor antagonists. Neuroendocrinology 83: 264-268, 2006.

33. Gatti L and Zunino F: Overview of tumor cell chemoresistance mechanisms. Methods Mol Med 111: 127-148, 2005.

34. Coley HM: Mechanisms and strategies to overcome chemotherapy resistance in metastatic breast cancer. Cancer Treat Rev 34: 378-390, 2008

35. Luqmani YA: Mechanisms of drug resistance in cancer chemotherapy. Med Princ Pract 14 (Suppl 1): 35-48, 2005.

36. Martinez-Lacaci I, Garcia Morales P, Soto JL and Saceda M Tumour cell resistance in cancer therapy. Clin Transl Oncol 9: 13-20, 2007.

37. Sekine I, Sasaki Y, Fujii H, et al: Recurrent breast cancer treated successfully with mitomycin-C and vinblastine after failure of both doxorubicin-containing regimen and paclitaxel - a case report. Tohoku J Exp Med 178: 331-337, 1996.

38. Legha SS: A review of mitomycin regimens in advanced breast cancer therapy. Clin Ther 7: 286-307, 1985.

39. Pasterz RB, Buzdar AU, Hortobagyi GN and Blumenschein GR: Mitomycin in metastatic breast cancer refractory to hormonal and combination chemotherapy. Cancer 56: 2381-2384, 1985.

40. Garewal HS: Mitomycin C in the chemotherapy of advanced breast cancer. Semin Oncol 15: 74-79, 1988.

41. Yusa K, Sato W, Yamazaki A, Tsukahara S and Tsuruo T: Crossresistance of human multidrug-resistant cells to mitomycin $\mathrm{C}$. Anticancer Res 11: 1301-1304, 1991.

42. Zhu Z, Mukhina S, Zhu T, Mertani HC, Lee K-O and Lobie PE: p44/42 MAP kinase-dependent regulation of catalase by autocrine human growth hormone protects human mammary carcinoma cells from oxidative stress-induced apoptosis. Oncogene 24 : 3774-3785, 2005.

43. Funakoshi T, Yanai A, Shinoda K, Kawano MM and Mizukami Y: $\mathrm{G}$ protein-coupled receptor 30 is an estrogen receptor in the plasma membrane. Biochem Biophys Res Commun 346: 904-910, 2006.

44. Vivacqua A, Bonofiglio D, Recchia AG, et al: The G proteincoupled receptor GPR30 mediates the proliferative effects induced by 17 beta-estradiol and hydroxytamoxifen in endometrial cancer cells. Mol Endocrinol 20: 631-646, 2006. 